\title{
The Alfvén resonator revisited
}

\author{
A. M. Hamza and W. Lyatsky \\ Physics Department, University of New Brunswick, Canada
}

Received: 19 October 2009 - Revised: 17 January 2010 - Accepted: 21 January 2010 - Published: 2 February 2010

\begin{abstract}
Two models for a magnetosphere-ionosphere coupling feedback instability in the lower magnetosphere are studied. In both models the instability arises because of the generation of an Alfvén wave from growing arc-like structures in the ionospheric conductivity. The first model is based on the modulation of precipitating electrons by field-aligned currents of the upward moving Alfvén wave (Modulation Model). The second model takes into consideration the reflection of the Alfvén wave from a maximum of the Alfvén velocity at about $3000 \mathrm{~km}$ altitude (Reflection Model). The growth of structures in both models takes place when the ionization function associated with upward field aligned current is shifted from the edges of enhanced conductivity structures to their centers. Such a shift arises because the structures move along the ionosphere at a velocity different from the $\boldsymbol{E} \times \boldsymbol{B}$ drift velocity. As a result, field-aligned currents of upward propagating Alfvén wave at some altitude appear shifted with respect to the edges of the structures. Although both models may work, the growth rate for the first model, as based on the modulation of the precipitating accelerated electrons, for typical conditions, may be tens or more times larger than that for the second model based on the Alfvén wave reflection. The proposed models can provide the growth of both single and periodic structures. When applied to auroral arc generation the studied instability leads to high growth rates and narrow arcs. The physical mechanism is mostly suitable for the generation of auroral arcs with widths of the order of $1 \mathrm{~km}$ and less. The growth rate of the instability for such structures can be as large as $0.3 \mathrm{~s}^{-1}$. In the case of periodic structures, their motion must lead to the generation of magnetic pulsations with periods of about $1-6 \mathrm{~s}$, which is close to the expected period of Alfvén resonant oscillations in the lower magnetosphere. However, these oscillations (for the first and most effective model MM) are
\end{abstract}

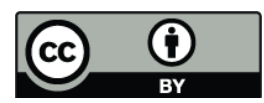

Correspondence to: A. M. Hamza (ahamza@unb.ca) not exactly Alfvén resonant oscillations. These oscillations are modulations in the ionospheric density, which propagate along the ionospheric currents and not along the magnetic field lines.

Keywords. Ionosphere (Plasma waves and instabilities)

\section{Introduction}

It has been shown that the magnetospheric convection can be unstable and may be divided into convection streams. Several causes for such convection separation have been suggested: The interchange or shear flow instability (e.g., Roux, 1996; Samson et al., 1996; Voronkov et al., 1997), the ionospheric feedback instability (e.g., Sato and Holzer, 1973; Leontyev and Lyatsky, 1982; Trakhtengertz and Feldstein, 1984; Watanabe and Sato, 1988; Lysak, 1991), and the magnetosphere-ionosphere coupling instability including an active role of magnetospheric plasma and taking into consideration the effect of field-aligned currents on the ionospheric conductivity (Kozlovsky and Lyatsky, 1994, 1999). These models suggest that the convection streams arising as a result of this instability may be responsible for the generation of auroral arcs. An important role of magnetosphere-ionosphere coupling in auroral arc generation was recently demonstrated by Newell et al. (1996a, b) who showed a strong suppression of aurora in the sunlit ionosphere.

There are two versions of the magnetosphere-ionosphere coupling feedback instability. One version is related to the excitation of entire magnetic field lines from one ionosphere to the other (Sato and Holzer, 1973; Kozlovsky and Lyatsky, 1994, 1999). The second version is related to the development of the instability in the lower magnetosphere (e.g., Leontyev and Lyatsky, 1982; Trakhtengertz and Feldstein, 1984; Lysak, 1991). This version of the instability is based on the generation of an Alfvén wave by growing arclike structures in the ionospheric conductivity. Two different 
models of the magnetosphere-ionosphere coupling feedback instability in the lower magnetosphere have been proposed. One model (e.g., Leontyev and Lyatsky, 1982) (the Modulation Model MM from here on) is based on the modulation of precipitating electrons by field-aligned currents of the upward moving Alfvén wave. The other model (Trakhtengertz and Feldstein, 1984; Lysak, 1991) (the Reflection Model RM from here on) takes into consideration the reflection of the Alfvén wave from a maximum of the Alfvén velocity at about $3000 \mathrm{~km}$ altitude. The growth of structures in both models takes place when the ionization function associated with upward field aligned current is shifted from the edges of enhanced conductivity structures to their centers.

The aim of the present paper is to continue the study of this instability and to compare the efficiency of the two models (as based on the modulation of precipitating electrons by field-aligned currents of the Alfvén wave and on the reflection of the Alfvén wave at an Alfvén velocity maximum at about $3000 \mathrm{~km}$, respectively) for a magnetosphereionosphere coupling feedback instability in the lower magnetosphere.

\section{Model description and problem solution}

A self-consistent model for the stratification of magnetospheric convection was first proposed by Sato and Holzer (1973), who showed that upward field-aligned currents of an Alfvén wave reflected from the conjugate ionosphere may lead to an additional increase in ionization inside the growing structures, and consequently to the development of the instability. This model as well as that proposed by Kozlovsky and Lyatsky $(1994,1999)$ consider the instability in the global magnetosphere-ionosphere system including both conjugate ionospheres. A self-consistent model for the stratification of the magnetospheric convection in the lower magnetosphere was first proposed by Leontyev and Lyatsky (1982), who showed that upward field-aligned currents of Alfvén waves generated by growing arc-like structures in the ionospheric conductivity can lead to the modulation of precipitating electrons and to an ionospheric feedback instability in the lower ionosphere. The problem was solved numerically and it was shown that the instability can indeed take place.

The recent experimental results published by Newell et al. (1996a, b) have stimulated our interest in this very fundamental field of research and led us to study the problem in some more details. We will derive the dispersion equation for the instability, examine the growth rate of the generated structures, and compare the growth rate of the instability to that given by another possible model for the stratification of the magnetospheric convection in the lower magnetosphere to be discussed in the next section.

To study this problem we will follow some of the steps in Leontyev and Lyatsky (1982), and will suggest the existence of a region of field-aligned electron acceleration at an altitude of about $1 R_{\mathrm{E}}$ (e.g., Olson et al., 1996). We will also assume that the field-aligned currents associated with an Alfvén wave generated by an ionospheric inhomogeneity, traveling in this acceleration region, produce an additional downward electron acceleration in the region of upward field-aligned current, and an electron braking or deceleration in the region of downward current. The growth of structures takes place when the ionization function associated with upward field aligned current is shifted from the edges of enhanced conductivity structures to their centers. Such a shift arises because the structures move along the ionosphere at a velocity different from the $\boldsymbol{E} \times \boldsymbol{B}$ drift velocity. As a result, field-aligned currents of upward propagating Alfvén waves, at some altitude, appear shifted with respect to the edges of the structures as shown in Fig. 1a.

We proceed and use the continuity equation to evaluate the field-aligned currents

$j_{\mathrm{Z}}=\nabla_{\perp} \cdot \boldsymbol{J}=\nabla_{\perp} \cdot\left(\Sigma_{\mathrm{P}} \boldsymbol{E}+\Sigma_{\mathrm{H}} \hat{\boldsymbol{b}} \times \boldsymbol{E}\right)$

where $j_{\mathrm{Z}}$ is the field-aligned current (positive direction is along the magnetic field), $\boldsymbol{J}$ is the height-integrated ionospheric current, $\Sigma_{\mathrm{P}}$ and $\Sigma_{\mathrm{H}}$ are the height-integrated Pedersen and Hall ionospheric conductivities, $\boldsymbol{E}$ is the electric field, and $\hat{b}$ is the unit vector along the magnetic field. The magnitude of field-aligned currents in an Alfvén wave propagating in the magnetosphere can be derived as follows (Maltsev et al., 1977; Leontyev and Lyatsky, 1982; Lysak, 1991),

$j_{\mathrm{z}}=-\nabla_{\perp} \cdot \boldsymbol{J}_{\mathrm{m}}=-\Sigma_{\mathrm{w}} \frac{\partial E^{\mathrm{up}}}{\partial x}$

where $\boldsymbol{J}_{\mathrm{m}}$ are transverse magnetospheric currents in the Alfvén wave, closing field-aligned currents, $\boldsymbol{E}^{\text {up }}$ is the transverse electric field of the upward moving Alfvén wave, $\Sigma_{\mathrm{w}}$ is the magnetospheric wave conductivity

$\Sigma_{\mathrm{w}}=\frac{c^{2}}{4 \pi V_{\mathrm{A}}}$

where $c$ is the light speed and $V_{\mathrm{A}}$ is the Alfvén speed. The average value of the plasma density in the E-region is derived from the ionization balance equation for electrons:

$\frac{\partial n}{\partial t}+\frac{\partial}{\partial x}\left(n v_{\mathrm{x}}\right)=q-\alpha n^{2}$

where $v_{\mathrm{x}}$ is the x-component of the $\boldsymbol{E} \times \boldsymbol{B}$ drift velocity, $q$ is the ionization function, and $\alpha$ is the recombination factor. Assuming the ionization function to be proportional to the magnitude of upward field-aligned current $j_{z, \mathrm{~A}}$ at point A of the acceleration region just over the point $x$ where we derive the electron density (see Fig. 1), we can write

$q=\chi a j_{\mathrm{Z}, \mathrm{A}}(x, t)=\chi a j_{\mathrm{Z}}\left(x-v_{\mathrm{X}} \delta t, t-\delta t\right)$

where $j_{\mathrm{Z}}\left(t-\delta t, x-v_{\mathrm{x}} \delta t\right)$ is the field-aligned current over the ionosphere level at time $t-\delta t$ and for the coordinate $x-$ $v_{\mathrm{X}} \delta t$. It is necessary to evaluate the field-aligned current at 
(a)
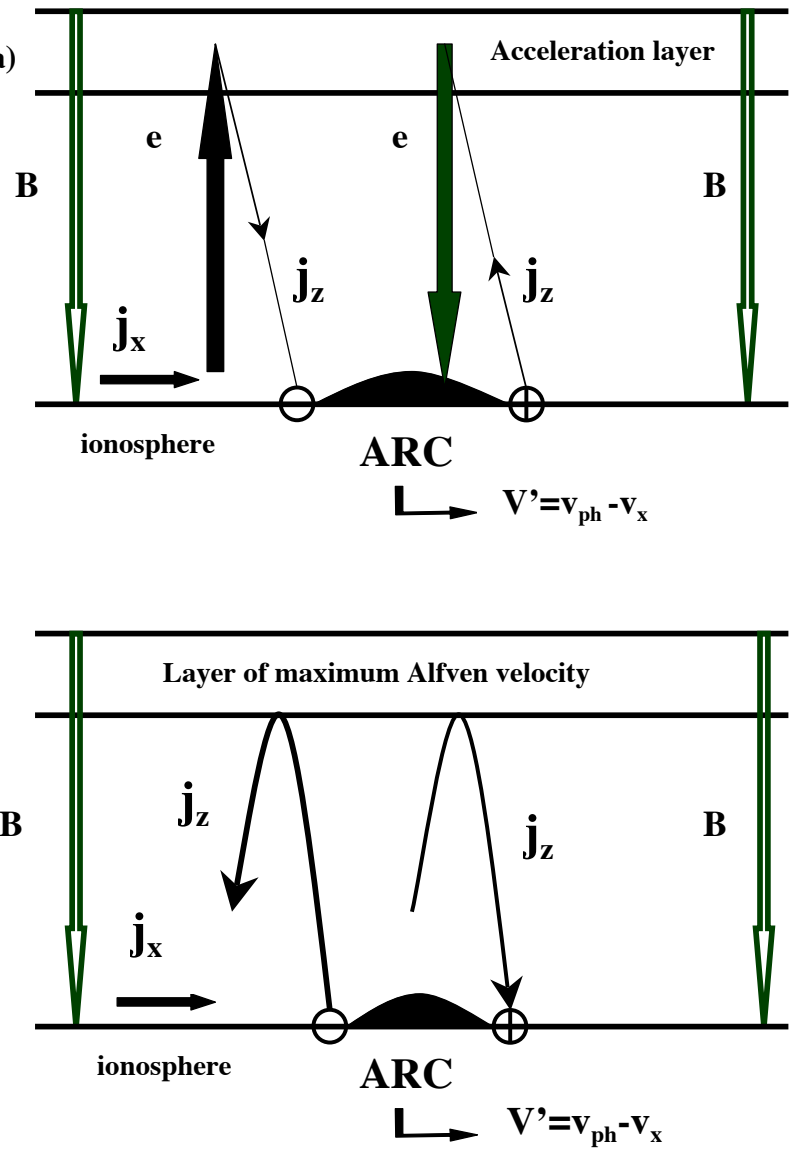

Fig. 1. A scheme explaining the growth of a strip of enhanced ionospheric conductivity, which is thought to be an auroral arc, for two magnetosphere-ionosphere coupling feedback instability models believed to occur in the lower magnetosphere. The instability arises because field-aligned currents $j_{\mathrm{Z}}$ of an Alfvén wave emitted by the arc-like structure are generated. The first model (a) is based on the modulation of precipitating electrons by field-aligned currents of the upward moving Alfvén wave (the additional accelerated electrons are shown by the symbol "e"). The second model (b) takes into consideration the reflection of the Alfvén wave from a maximum of the Alfvén velocity located at altitude of about $3000 \mathrm{~km}$. The growth of arc-like structures takes place when the ionization function is shifted from the edges of enhanced conductivity structures to their centers. Such a shift arises when the structure moves along the ionosphere at a velocity $v_{\mathrm{ph}}$, different from the $v_{\mathrm{x}}=c E_{\mathrm{y}} / B$ drift velocity. As a result, the field-aligned currents of the Alfvén wave are shifted relatively to the edges of the structures, which leads to a shift of the ionization function.

an ulterior time in order to take into account the time delay $\delta t$ and spatial shift $v_{\mathrm{x}} \delta t$ required for the wave to be exited in the ionosphere and to reach the acceleration region at moment $t$ at point $\mathrm{A}$, as shown in Fig. 1 . The time delay $\delta t$ is equal to $z_{1} / V_{\mathrm{A}}$, where $z_{1} \approx 1 R_{\mathrm{E}}$ is the altitude of the acceleration layer. The factor $\chi$ in Eq. (5) is the efficiency of ionization produced by one precipitating electron, which can be written in the following form:

$\chi=1+\delta \frac{\epsilon}{\epsilon_{\mathrm{i}}}$

where $\epsilon$ is the energy of precipitating electrons in $\mathrm{eV}, \epsilon_{\mathrm{i}}$ is the ionization potential $(\sim 16 \mathrm{eV})$ and the factor $\delta \approx 2$. (e.g., Kozlovsky and Lyatsky, 1994, 1999; Lyatsky, 1999). The factor $a$ in Eq. (5) is given by:

$a=\frac{1}{e \Delta z}$

where $e$ is the electron charge, and $\Delta z$ is the thickness of the E-region. In the linear approximation, using a plane wave $\exp (-i \omega t+i \mathbf{k} \cdot \mathbf{r})$ analysis, and assuming $\delta \Sigma_{\mathrm{P}} / \delta \Sigma_{\mathrm{P} 0}=\delta n / n_{0}$, where the subscript 0 means undisturbed values, we obtain from expressions (1-7) the following expression for the dispersion equation

$\omega-\boldsymbol{k} \cdot \boldsymbol{v}+i 2 \alpha n_{0}=\boldsymbol{k} \cdot \boldsymbol{A} \exp i(\omega-\boldsymbol{k} \cdot \boldsymbol{v}) t$

where

$\boldsymbol{A}=\chi \frac{a}{n_{0}} \frac{\boldsymbol{J}_{\mathrm{eff}}}{1+\frac{\Sigma_{\mathrm{p}}}{\Sigma_{\mathrm{w}}}}$

where $\boldsymbol{J}_{\text {eff }}=\Sigma_{\mathrm{P}}\left[\boldsymbol{E}+\left(\delta \Sigma_{\mathrm{H}} / \delta \Sigma_{\mathrm{P}}\right) \hat{\boldsymbol{b}} \times \boldsymbol{E}\right]$. When the Hall to Pedersen conductivity ratio $\delta \Sigma_{\mathrm{H}} / \delta \Sigma_{\mathrm{P}} \approx \Sigma_{\mathrm{H}} / \Sigma_{\mathrm{P}}$, the effective current $\boldsymbol{J}_{\text {eff }}$ is equal to the ionospheric current.

The dispersion relation (8) can be separated into real and imaginary parts, allowing us to evaluate the real part of the frequency and the growth rate.

$\tilde{\omega}=\boldsymbol{k} \cdot \boldsymbol{A} \cos (\tilde{\omega} \delta t)$

$\gamma+2 \alpha n_{0}=\boldsymbol{k} \cdot \boldsymbol{A} \sin (\tilde{\omega} \delta t)$

where $\tilde{\omega}=\operatorname{Re}(\omega-\boldsymbol{k} \cdot \boldsymbol{v})=k\left(v_{\mathrm{ph}}-v_{\mathrm{x}}\right)$. From Eq. (11) we have that for $\boldsymbol{k} \cdot \boldsymbol{A}>0$ the growth rate $\gamma$ is maximum when

$\tilde{\omega} \delta t \approx\left(\frac{\pi}{2}-\epsilon+2 m \pi\right)$
$\tilde{\omega} \delta t \approx\left(\frac{3 \pi}{2}-\epsilon+2 m \pi\right)$

where $\epsilon$ is a small value $\epsilon \ll 1$, and $m=0,1,2,3, \ldots$ The upper expression is related to $\tilde{\omega}>0$ when $\left(v_{\mathrm{ph}}-v_{\mathrm{x}}\right)>0$; the lower expression is related to $\tilde{\omega}>0$ when $\left(v_{\mathrm{ph}}-v_{\mathrm{x}}\right)<0$. For negative $\boldsymbol{k} \cdot \boldsymbol{A}<0$ we have the opposite situation. The dependence of the wave vector $\boldsymbol{k}$ and growth rate $\gamma$ on $\tilde{\omega} \delta t$ is illustrated in Fig. 2.

For the case of maximum growth rate, $\cos \tilde{\omega} \delta t \approx \epsilon$ and $\sin \tilde{\omega} \delta t \approx 1$, and for the most important case $\tilde{\omega} \delta t \approx$ $(\pi / 2-\epsilon+2 m \pi)>0$ we obtain from Eq. (11)

$$
\begin{aligned}
& k \approx \frac{\frac{\pi}{2}+2 m \pi}{\epsilon A \delta t}<k_{\max } \\
& \gamma+2 \alpha n_{0} \approx \boldsymbol{k} \cdot \boldsymbol{A}
\end{aligned}
$$



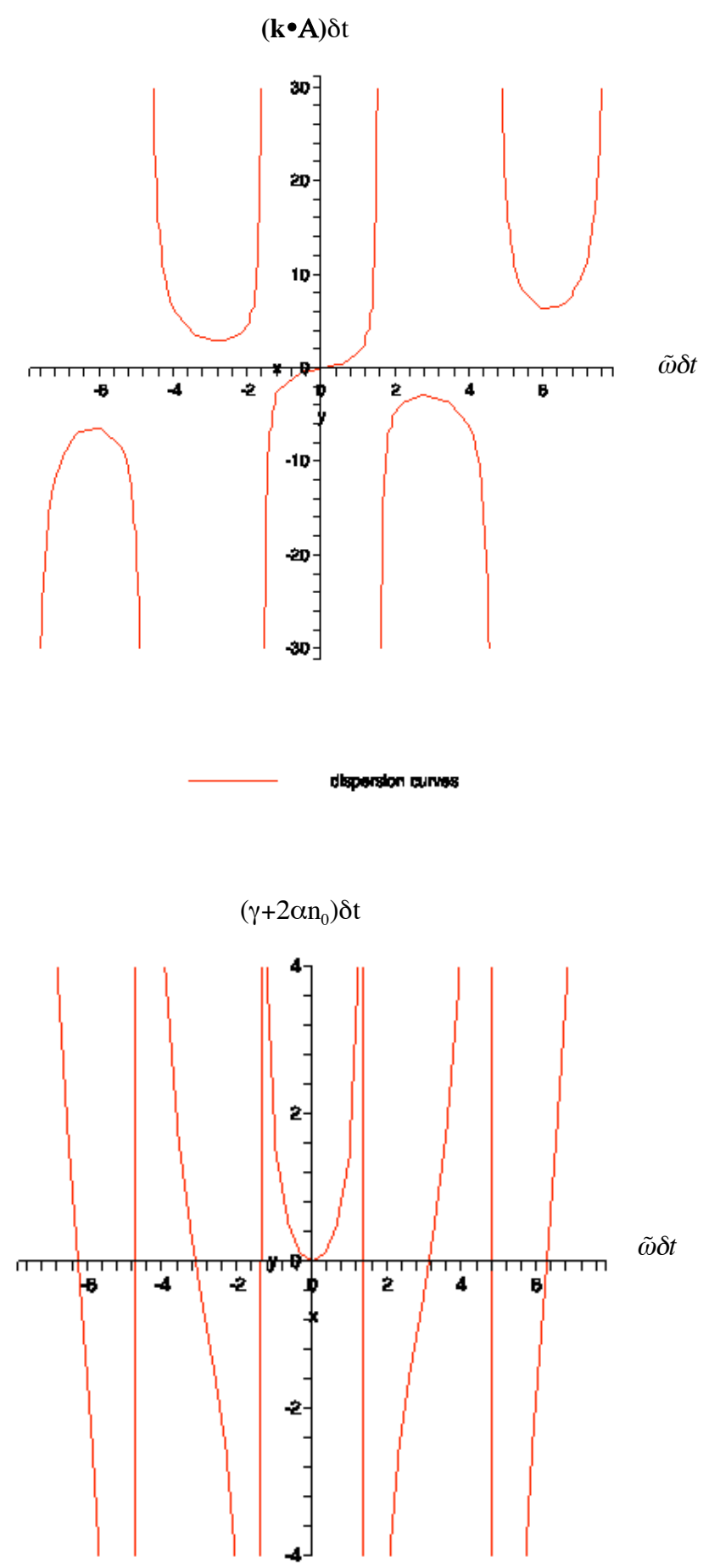

Fig. 2. The solution of the dispersion equation. Upper panel shows the dependence of the $\boldsymbol{k}$ vector versus $\tilde{\omega} \delta t$, where $\tilde{\omega}$ is the frequency in the coordinate system moving at the $\boldsymbol{E} \times \boldsymbol{B}$ drift velocity. The lower panel shows the dependence of the growth rate $\gamma$ versus $\tilde{\omega} \delta t$ units.
The magnitude $k$ in Eq. (13) cannot be larger than $k_{\max }$, which corresponds to a minimum size for the generated structures, which is larger than some Larmor radius of accelerated electrons, which is of the order of few hundreds of meters. We can then rewrite Eq. (13) for $\epsilon$

$\frac{\frac{\pi}{2}+2 m \pi}{k_{\max } A \delta t}<\epsilon \ll 1$

We now estimate the magnitude of $\epsilon$ as given by Eq. (14). For typical magnitudes $\chi=30, \Delta z=20 \mathrm{~km}, n=5 \times 10^{11} \mathrm{~m}^{-3}$, and $\boldsymbol{J}_{\text {eff }}=0.1 \mathrm{~A} \mathrm{~m}^{-1}$ and $\Sigma_{\mathrm{P}} / \Sigma_{w}=5$ (e.g., Kozlovsky and Lyatsky, 1999; Lyatsky, 1999), we obtain $A \approx 0.3 \mathrm{~km} \mathrm{~s}^{-1}$. The magnitude of $\delta t$ is equal to the time of propagation of an Alfvén wave to the acceleration layer, that is about $3 \mathrm{~s}$. Substituting these magnitudes into Eq. (14) and assuming $k_{\max }^{-1} \approx 1 \mathrm{~km}$ yields

$m+\frac{1}{4}<\epsilon<1$

This expression is satisfied for $m=0$ but not for larger $m$. This means that it is sufficient to restrict ourselves only to the case $m=0$, though for some conditions the excitation of waves with larger $m$ may be possible as well. We also note that in the case of a phase shift close to $\pi / 2$, a single auroral arc can be excited because the additional ionization comes down to the same structure. In the case of larger phase shifts it is impossible to explain single arcs but only multiple periodic structures.

Thus the most probable scenario is the excitation of waves propagating along the ionospheric current. Because the growth rate is proportional to the wave vector $\boldsymbol{k}$, this instability should lead to the excitation of very narrow structures with sizes as small as $1 \mathrm{~km}$ and even less. The phase velocity of these structures is close to the drift velocity of the plasma. The magnitude of the growth rate can be estimated by substituting in Eq. (14) the typical value of $A=0.3 \mathrm{~km} / \mathrm{s}$ as obtained earlier. Then, we obtain $\gamma \approx 300 k$. Assuming $k=10^{-3} \mathrm{~m}^{-1}$ yields $\gamma \approx 0.3 \mathrm{~s}^{-1}$.

We note that the motion of the periodic structures at speeds close to the $\boldsymbol{E} \times \boldsymbol{B}$ drift must lead to observations of magnetic pulsations. If the wave length is $\lambda=2 \pi / k \approx 6 \mathrm{~km}$ and the drift velocity is about $1 \mathrm{~km} \mathrm{~s}^{-1}$ the pulsation period is about $6 \mathrm{~s}$. This is close to the expected period of the Alfvén resonant oscillations in the lower magnetosphere. However, these oscillations are not exactly Alfvén resonant oscillations as we will see in the next section. These structures arise as modulations of the ionospheric conductivity that propagate along the ionospheric currents and not along the magnetic field lines. They are ionospheric structures, which can trigger the launch of Alfvén waves as we have suggested.

\section{Alfvén resonance excitation model}

Another possible model for the stratification of the magnetospheric convection and the excitation of arc-like structures in 
the lower magnetosphere was proposed by Trakhtengertz and Feldstein (1984) and Lysak (1991). This model is based on the reflection of Alfvén waves (RM), emitted from the ionospheric inhomogeneities, from a region of enhanced Alfvén velocity at an altitude of about $3000 \mathrm{~km}$ often called the Dessler maximum in the Alfvén velocity. This model is in fact the model of Sato and Holzer (1973) applied to the lower magnetosphere.

The scheme explaining this instability is shown in Fig. $1 \mathrm{~b}$. The necessary shift of resulting field-aligned currents from the edges of the structures is provided by field-aligned currents of the reflected Alfvén wave shifted along the ionosphere due to the motion of the structures. The dispersion equation for this instability can be obtained using the same method used earlier, but some equations must be exchanged. The equation describing the field-aligned currents of the reflected Alfvén wave need to be added. The equation is similar to Eq. (2) but holds a minus sign in front.

$j_{\mathrm{z}}=\nabla_{\perp} \cdot \boldsymbol{J}_{\mathrm{m}}=\Sigma_{\mathrm{w}} \frac{\partial E^{\mathrm{up}}}{\partial x}$

The total disturbed electric field is now a sum of both upward and downward wave electric field $\delta \boldsymbol{E}=\boldsymbol{E}^{\mathrm{up}}+\boldsymbol{E}^{\text {down }}$. The equation for ionization balance is the same, but the ionization function $q$ must be written now in some other form with the time delay being twice as long in order to take into account the time delay required for the reflected wave to reach the ionosphere (see Fig. 1b). The time delay $\delta t$ in Eq. (5) is equal now to $2 z_{2} / V_{\mathrm{A}}$ where $z_{2}$ is the altitude of the Dessler maximum. For simplicity we consider a simplified model suggesting that the wave is reflected from a narrow boundary at an altitude of about $3000 \mathrm{~km}$. The simplified model we have just presented neglects some important aspects related to the profile of the Alfvén velocity. The earlier models of Trakhtengertz and Feldstein (1984) and Lysak (1991) used an exponential profile of the plasma density that allowed them to find, in the second case, some closed form solutions and perform some numerical simulations. The present approach allows to obtain the dispersion relation and estimates the growth rate. We have retained all the physics that was included in the previous models mentioned above.

The last important feature of this model is that the factor $\chi$ in expression (5) (for the ionization function), the efficiency of ionization produced by one precipitating electron, is now equal to 1 because the Alfvén reflection model does not take into consideration accelerated electrons. The acceleration takes place rather far from the ionosphere, and accelerated electrons do not follow the reflected Alfvén wave. Taking into account a modification of electron acceleration by Alfvén waves leads us immediately to the previous model described above. The variation of the ionospheric conductivity in this model (RM) is produced not by accelerated electrons but by cold particles carrying field-aligned currents of Alfvén waves. In the linear approximation, using a plane wave $\exp (-i \omega t+i \boldsymbol{k} \cdot \boldsymbol{r})$ analysis, and from Eqs. (1-6) and (17) we obtain the following dispersion equation

$\omega-\boldsymbol{k} \cdot \boldsymbol{v}+2 i \alpha n_{0}=-\frac{a}{n_{0}} \frac{\boldsymbol{k} \cdot \boldsymbol{J}_{\mathrm{eff}}}{1+\frac{\Sigma_{\mathrm{P}}}{\Sigma_{\mathrm{w}}} \frac{1+R e^{i \phi}}{1-R e^{i \phi}}}$

where $R$ is the reflection factor for the Alfvén wave and $\phi=$ $\tilde{\omega} \delta t$, where again $\tilde{\omega}=\operatorname{Re}(\omega-\boldsymbol{k} \cdot \boldsymbol{v})$. The expression (18) is separated into a real and an imaginary part as follows:

$\omega_{r}=\operatorname{Re}(\omega)=\boldsymbol{k} \cdot \boldsymbol{V}_{0}+\boldsymbol{k} \cdot \boldsymbol{A}_{1}\left(1+\frac{\Sigma_{\mathrm{P}}}{\Sigma_{\mathrm{w}}} \frac{1-R^{2}}{\left|1-R e^{i \phi}\right|^{2}}\right)$

$\gamma=-\boldsymbol{k} \cdot \boldsymbol{A}_{1} \frac{\Sigma_{\mathrm{P}}}{\Sigma_{\mathrm{w}}} \frac{2 R \sin \phi}{\left|1-R e^{i \phi}\right|^{2}}-2 \alpha n_{0}$

where

$\mathbf{A}_{1}=-\frac{a}{n_{0}} \frac{\mathbf{J}_{\mathrm{eff}}}{\left|1+\frac{\Sigma_{\mathrm{P}}}{\Sigma_{\mathrm{w}}} \frac{1+R e^{i \phi}}{1-R e^{i \phi}}\right|^{2}}$

The magnitude of the growth rate is maximum for $\phi=\tilde{\omega} \delta t \approx$ $\pi / 2+2 m \pi$, where $m=0,1,2,3, \ldots$ In this case the real and imaginary parts of $\omega=\omega_{r}+i \gamma$ can be rewritten as follows:

$$
\begin{aligned}
& \omega_{r}=\boldsymbol{k} \cdot \boldsymbol{V}_{0}+\boldsymbol{k} \cdot \boldsymbol{A}_{1}\left(1+\frac{\Sigma_{\mathrm{P}}}{\Sigma_{\mathrm{w}}} \frac{1-R^{2}}{1+R^{2}}\right) \\
& \gamma=-\boldsymbol{k} \cdot \boldsymbol{A}_{1} \frac{\Sigma_{\mathrm{P}}}{\Sigma_{\mathrm{w}}} \frac{2 R}{1+R^{2}}-2 \alpha n_{0} \\
& \boldsymbol{A}_{1}=-\frac{a}{n_{0}} \frac{\boldsymbol{J}_{\mathrm{eff}}}{1+\left(\frac{\Sigma_{\mathrm{P}}}{\Sigma_{\mathrm{w}}}\right)^{2}+\left(\frac{\Sigma_{\mathrm{P}}}{\Sigma_{\mathrm{w}}} \frac{1-R^{2}}{1+R^{2}}\right)^{2}}
\end{aligned}
$$

The maximum value for the growth rate taking place for $R=$ 1 is given by

$\gamma_{R=1}=-\frac{a}{n_{0}} \boldsymbol{k} \cdot \boldsymbol{J}_{\mathrm{eff}} \frac{\frac{\Sigma_{\mathrm{P}}}{\Sigma_{\mathrm{w}}}}{1+\left(\frac{\Sigma_{\mathrm{P}}}{\Sigma_{\mathrm{w}}}\right)^{2}}-2 \alpha n_{0}$

Taking typical magnitudes for the different quantities in expression (25) and for a ratio $\Sigma_{\mathrm{P}} / \Sigma_{\mathrm{W}} \approx 5$, the maximum magnitude for the growth rate appears to be $\chi$ (ionization efficiency) times less than that given by Eq. (14) for the previous model (the modulation model MM). For more realistic conditions when the reflection coefficient is less than 1 , the growth rate becomes smaller. Since the $\chi$ factor is about 30 , the Alfvén resonator excitation model (RM) is also 30 times less effective than the model taking into consideration the modulation of electron acceleration by field-aligned currents of the Alfvén waves (MM), considered above. Nevertheless, for some situations for which the magnitude of the ratio $\Sigma_{\mathrm{P}} / \Sigma_{\mathrm{W}}$ is small, this mechanism can also work. 
(a)

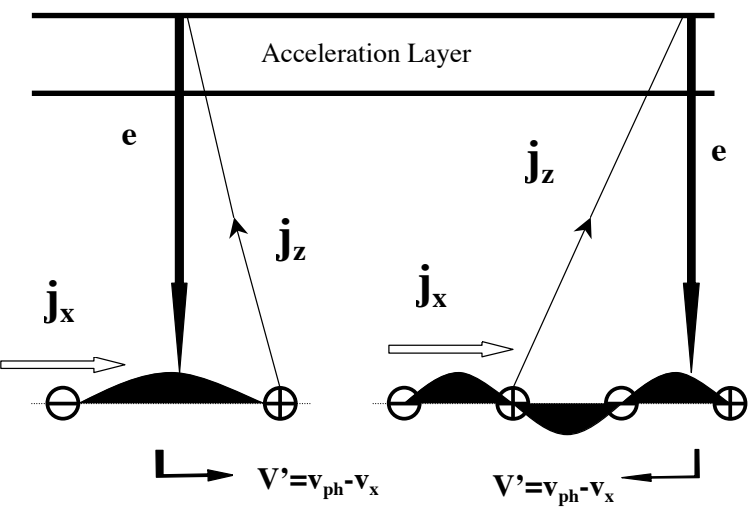

(b)

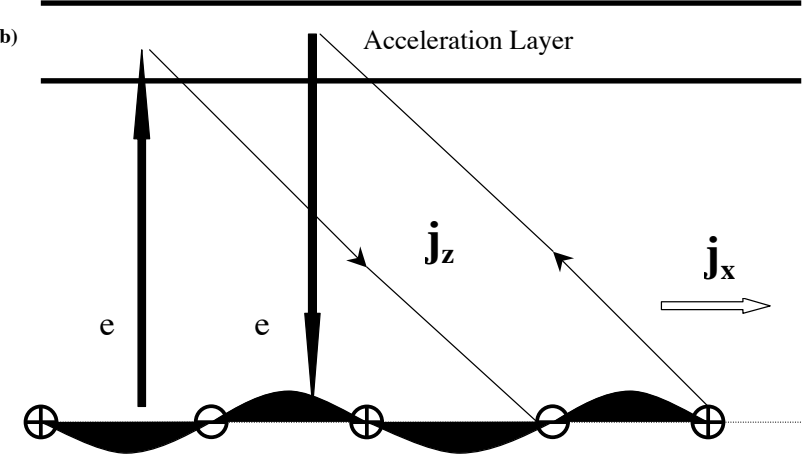

Fig. 3. A scheme explaining the generation of a single arc-like structure (a), and multiple periodic structures (b). $v^{\prime}=v_{\mathrm{ph}}-v_{\mathrm{X}}$ is the relative velocity of the structures in the coordinate system moving at the $\boldsymbol{E} \times \boldsymbol{B}$ drift velocity.

\section{Discussion}

Two models describing the modification of convection in the lower magnetosphere and its separation or break up into narrow streams are considered. Both models include the generation of Alfvén waves by ionospheric inhomogeneities. The first model (MM), first proposed by Leontyev and Lyatsky (1982), is based on the modulation of precipitating electrons by field-aligned currents of the upward Alfvén wave. The second model (RM), first proposed by Trakhtengertz and Feldstein (1984) is a modification of the original Sato and Holzer (1973) model; this model (RM) takes into consideration the reflection of Alfvén wave from a maximum in the Alfvén velocity at about $3000 \mathrm{~km}$ altitude. The growth of waves in both models takes place when the ionization function associated with upward field aligned currents appears to be shifted from the edges of structures toward their centers. Such a shift can take place because the arising structures move along the ionosphere at some velocity which is not necessarily equal to the drift velocity. As a result, field-aligned currents of the upward propagating Alfvén wave at some altitude appear shifted with respect to the edges of the struc- tures. If the upward field-aligned current is shifted toward the center of the ionospheric strip, the latter grows. Estimates show that the growth rate of the first instability as based on the modulation of the precipitating accelerated particles by field-aligned currents of the Alfvén wave, for typical conditions, is expected to be tens or more times larger than that for the second instability as based on the Alfvén wave reflection from the Dessler maximum in the Alfvén velocity. This is because the first mechanism is thought to be associated with the modulation of accelerated electrons whereas the second mechanism is suitable only for cold electrons which have a small ionization efficiency. We should point out that this reflection model (RM) cannot be associated with hot electrons because they are accelerated rather far from the ionosphere and do not follow the field-aligned currents of the Alfvén wave as taken at the ionospheric level.

Both proposed models can lead to the development of both single and periodic structures. Single structures can take place when the phase shift between the original upward fieldaligned current of the Alfvén wave and the ionization function is less than $\pi$, so that the additional ionization leads to an increase in conductivity inside the same structure. When the phase shift is larger than $\pi$, it can lead to the development of multiple periodic structures. The formation of both single and multiple structures is demonstrated in Fig. 3. Returning to the problem of auroral arc generation, we note that there are three important features of arcs, which must be explained. They are (e.g., Borovsky, 1993): a high growth rate of arc formation, narrowness of arcs, and their orientation close to the auroral oval. The proposed model (MM) enables us to explain the first two. The growth rate of this instability is proportional to the $\boldsymbol{k}$ vector, which means that the generation of narrow structures is most effective. Therefore this mechanism seems to be suitable for generation of very narrow auroral arcs with width of about $1 \mathrm{~km}$ and less. The growth rate of the instability for such narrow structures can be as large as $0.3 \mathrm{~s}^{-1}$. We note however, that this mechanism enables us to explain auroral arcs only in one ionosphere, and it is not suitable for excitation of wide and conjugate auroral arcs; such arcs are more likely excited not in the inner magnetosphere but along global magnetic field lines as considered by Kozlovsky and Lyatsky (1994, 1999). We note also that the proposed mechanism for auroral arc generation may be responsible for small-scale structure inside a wider arc. In this case, an original wide arc provides some original electron acceleration (an increase in the ionization efficiency $\chi$ factor) that provides favorable conditions for the convection flow instability in the lower magnetosphere, discussed in this paper.

The phase velocity of these structures is close to the $\boldsymbol{E} \times \boldsymbol{B}$ drift velocity. In the case of the generation of periodic structures, their $\boldsymbol{E} \times \boldsymbol{B}$ drift must lead to the generation of magnetic pulsations. For structure widths of the order of $1 \mathrm{~km}$ (or wave length $\lambda \approx 6 \mathrm{~km}$ ), a drift velocity of $1 \mathrm{~km} \mathrm{~s}^{-1}$ leads to a pulsation period of about $6 \mathrm{~s}$. This is close to the expected 
period of the Alfvén resonant oscillations in the lower magnetosphere. However, these oscillations (for the first most effective modulation model MM) are not exactly Alfvén resonant oscillations; they are not associated with resonant cavity modes. Therefore observations of similar oscillations in the lower magnetosphere and ionosphere (e.g., Boesinger et al., 1999) should not be interpreted as Alfvén resonant oscillations; we note that the authors cited above considered also an alternative interpretation of their results.

\section{Conclusions}

Two models for the stratification of the magnetospheric convection in the lower magnetosphere are considered. The first model is based on the modulation of precipitating electrons by field-aligned currents of an upward Alfvén wave emitted by growing ionospheric structures. A second model takes into consideration the reflection of Alfvén waves from an Alfvén velocity maximum layer located at about $3000 \mathrm{~km}$ altitude. The growth of conductivity structures in both models takes place when the ionization function associated with upward field aligned currents is shifted from the edges of enhanced conductivity structures toward their centers. Such a shift arises because the growing structures move along the ionosphere at a velocity different from the $\boldsymbol{E} \times \boldsymbol{B}$ drift velocity. As a result, field-aligned currents of upward propagating Alfvén wave at some altitude appear shifted with respect to the edges of the structures.

Estimates show that the growth rate of the first instability (MM) as based on the modulation of the precipitating accelerated electrons by field-aligned currents of the Alfvén wave, for typical conditions, may be tens or more times larger than that of the second instability based on the Alfvén wave reflection from the maximum in the Alfvén velocity. Both proposed models can explain the growth of both single and periodic structures. When applied to the auroral arc generation, this instability enables us to explain the high growth rate and the narrowness of auroral arcs. This mechanism is mostly suitable for generation of auroral arcs with widths of the order of less than $1 \mathrm{~km}$. The growth rate of the instability for such structures can be as large as $0.3 \mathrm{~s}^{-1}$.

We note however, that this mechanism enables us to explain auroral arcs developing only in one ionosphere, and it is unlikely to be suitable for the excitation of wide and conjugate auroral arcs; such arcs are more likely excited not in the inner magnetosphere but along global magnetic field lines as considered earlier by Kozlovsky and Lyatsky (1994, 1999). The proposed physical mechanism may be responsible for the formation of small-scale structures inside wider arc. In this case, an original wide arc provides some electron acceleration (an increase in the $\chi$ factor) that provides favorable conditions for the considered convection flow instability in the lower magnetosphere.
The phase velocity of the structures is close to the $\boldsymbol{E} \times \boldsymbol{B}$ drift velocity. In the case of the periodic structures, their motion must lead to the magnetic pulsation generation. For structures with wave lengths of order of $1-6 \mathrm{~km}$, a drift velocity of $1 \mathrm{~km} \mathrm{~s}^{-1}$ leads to a pulsation period of about $1-6 \mathrm{~s}$. This is close to the expected period of the Alfvén resonant oscillations in the lower magnetosphere. However, these oscillations (for the first most effective model) are not exactly real Alfvén resonant oscillations.

Acknowledgements. Funding for this study was provided by the Canadian Natural Science and Engineering Research Council (NSERC). This work was supported also by the Russian Foundation for Basic Research (grant 97-05-65894).

Topical Editor I. A. Daglis thanks P. Newell for his help in evaluating this paper.

\section{References}

Boesinger, T., Belyaev, P. P., and Rietveld, M. T.: Indications of ionospheric Alfvén resonator effects in spectra of ionospheric heating induced magnetic disturbances, Books of abstracts of the 9th Intern. EISCAT Workshop, Wernigerode, Germany, 610 September 1999, Max-Planck Institut fur Aeronomie, p.11, 1999.

Borovsky, J. E.: Auroral arc thickness as predicted by various theories, J. Geophys. Res., 98, 6101-6138, 1993.

Kozlovsky, A. E. and Lyatsky, W. B.: Instability of the magnetosphere-ionosphere convection and formation of auroral arcs, Ann. Geophys., 12, 636-641, 1994, http://www.ann-geophys.net/12/636/1994/.

Kozlovsky, A. and Lyatsky, W.: Finite Larmor radius convection instability in the near-Earth plasma sheet, J. Geophys. Res., 104, 2443-2449, 1999.

Leontyev, S. V. and Lyatsky, W. B.: Solitary auroral arc generation, Planet. Space Sci., 30, 1-4, 1982.

Lyatsky, W.: A possible role of ion demagnetization in substorm generation, J. Geophys. Res., 104, 19905-19910, 1999.

Lysak, R. L.: Feedback instability of the ionospheric resonant cavity, J. Geophys. Res., 96, 1553-1568, 1991.

Maltsev, Y. P., Lyatsky, W. B., and Lyatskaya, A. M.: Currents over the auroral arc, Planet. Space Sci., 25, 53-57, 1977.

Newell, P. T., Meng, C.-I., and Lyons, K. M.: Superpression of discrete aurora by sunlight, Nature, 381, 766-767, 1996a.

Newell, P. T., Lyons, K. M., and Meng, C.-I.: A large survey of electron acceleration events, J. Geophys. Res., 101, 2599-2614, 1996b.

Roux, A., Perraut, S., Robert, P., Morane, A., Pedersen, A., Korth, A., Kremser, G., Aparicio, B., Rodgers, D., and Pellinen, R.: Plasmasheet instability related to the westward traveling surge, J. Geophys. Res., 96, 17696-17714, 1991.

Samson, J. C., MacAulay, A. K., Rankin, R., Fricz, P., Voronkov, I., and Cogger, L. L.: Substorm intensifications and resistive flowballooning instabilities in the near-Earth magnetotail, in Proceedings of the Third International Conference on Substorm (ICS-3), Eur. Space Agency Spec. Publ. ESA SP-389, 399-404, 1996. 
Sato, T. and Holzer, T. E.: Quiet auroral arcs and electrodynamic coupling between the ionosphere and the magnetosphere, J. Geophys. Res., 78, 7314-7329, 1973.

Trakhtengertz, V. Y. and Feldstein, A. Y.: Quiet auroral arcs: Ionosphere effect of magnetospheric convection stratification, Planet. Space Sci., 32, 127-134, 1984.
Voronkov, I., Rankin, R., Fricz, P., Tikhonchuk, V. T., and Samson, J. C.: Coupling of shear flow and pressure gradient instabilities, J. Geophys. Res., 102, 9639-9650, 1997.

Watanabe, K. and Sato, T.: Self-excitation of auroral arcs in a threedimensional coupled magnetosphere-ionosphere system, Geophys. Res. Lett, 15, 717-720, 1988. 